\title{
Analysis of a Relationship Based Access Control Model
}

\author{
Phillipa Bennett \\ Computer Science \\ Department \\ Colorado State University \\ Fort Collins CO 80523-1873 \\ bennettp@cs.colostate.edu
}

\author{
Indrakshi Ray \\ Computer Science \\ Department \\ Colorado State University \\ Fort Collins CO 80523-1873 \\ iray@cs.colostate.edu
}

\author{
Robert France \\ Computer Science \\ Department \\ Fort Collins CO 80523-1873 \\ Colorado State University
}

\begin{abstract}
With the advent of online social networks (OSN) and personalized healthcare, relationship-based access control is playing an ever important role. People often share sensitive personal information through online social networks (OSNs) to keep in touch with their friends and families. Such sensitive information if leaked inadvertently to third parties may have detrimental effects and sometimes disastrous consequences on the lives of individuals. Access control models are needed that allows controlled sharing of information. Different features of the model may conflict with each other. Identifying potential conflicts is important as sometimes such conflicts are sources of misconfigurations. We propose an access control model for use in OSNs and provide its formal specification. Manually checking the formal specification is tedious and error-prone. Towards this end, we also demonstrate how conflicts and potential misconfigurations can be automatically detected using the Alloy Analyzer. We illustrate our approach using policies that are similar in spirit to those in use in existing OSNs.
\end{abstract}

\section{Categories and Subject Descriptors}

A.0 [General]: Conference proceedings; D.2.4 [Software /Program Verification]: Formal methods

\section{Keywords}

C3S2E, Access Control, Misconfigurations

\section{INTRODUCTION}

Online Social Networks (OSNs) are used for various purposes and impact society in many different ways. For example, on an individual level, they help families and friends separated by distance keep in touch and thus help mitigate undesirable effects of long distance relationships. Sensitive personal information is often shared through OSNs. On a

\footnotetext{
*Involved in the paper but now deceased
}

Permission to make digital or hard copies of part or all of this work for personal or classroom use is granted without fee provided that copies are not made or distributed for profit or commercial advantage and that copies bear this notice and the full citation on the first page. Copyrights for components of this work owned by others than ACM must be honored. Abstracting with credit is permitted. To copy otherwise, to republish, to post on servers or to redistribute to lists, requires prior specific permission and/or a fee. C3S2E' '15, July 13-15 2015, Yokohama, Japan Copyright 2015 ACM 978-1-4503-3419-8/15/-7 ...\$15.00. http://dx.doi.org/10.1145/2790798.2790811. broader level, social media contributed to the Arab Spring in 2011 and also helped President Obama raise $\$ 690 \mathrm{M}$ in his reelection campaign. Social media is used by a wide range of individuals and organizations, including the government, law-enforcement agencies, legitimate and rogue organizations, and different classes of individuals. Organizations have used the data generated in OSNs to do targeted advertising campaigns, develop new products, perform market research, trend analysis and reputation monitoring, and vet and hire human resources. Misuse of such sensitive information can have disastrous consequences on individuals such as damaged reputation, financial hardships, or compromised physical safety and security. In order to ensure the proper functioning of the social media and protect the confidentiality and integrity of the data shared through social networks, security and privacy issues must be addressed.

OSNs have evolved from simple networks of members sharing information to large networks whose differing types of relationships and interactions makes them difficult to understand and analyse. Researchers have proposed access control policies and models $[3,5,12,18]$ for OSNs. Relationshipbased access control policies for OSNs are based on the structure, and on different models of sharing $[2,7,6,11,21,20$, 22]. In relationship-based access control a member will give the right to access, or the right to perform an operation on, private objects they own to other members based on their direct and indirect relationships. Various researchers have proposed different factors that influence access control in OSNs. Fong et al. $[11,2]$ advocated that access of a subject to an object depends both on the relationship and the context of access. Crampton [8] associates permissions with principals and subjects issue request to perform certain operation on object. If the path from the subject to the object satisfies the condition(s) specified in the principal, then we check if the action is allowed by that principal. Carminati et al. [4] define permissions on the basis of type, depth, and trust level. Most of these works focus on factors that influence the access to the resource by an user.

OSN access control policies on the various artefacts are not necessarily independent, and interact in subtle ways that may give rise to inconsistencies and conflicts. In such cases, the default conflict resolution mechanisms decide which policies have higher priority. Thus, if a user has specified policies in an incorrect manner, his sensitive information may be leaked unintentionally. Researchers have worked on misconfiguration detection, that is, on uncovering situations in which the user's intent is inaccurately mapped to her access control settings $[14,15,16]$. Policy misconfigurations have 
also been explored in the context of general access control $[1,9,10,17,19]$ to categorize static permissions $[10,17]$, to identify dynamic/historical access patterns [1, 9, 19], to prioritize and quantify the impact of misconfigurations [9], and to conduct user studies to detect access control rule patterns to help configure the access control settings $[14,15,16]$.

In this paper, we begin by formalizing a unified relationshipbased access control model, ReBAC, that captures the essence of earlier proposed access control model. The novelty of our proposed model is that it explores the complex interdependencies between the various relationships, the different operations, and access privileges. Understanding such relationships is important in order to reason about access control. Such reasoning is important as the wide variety of artefacts that are shared by the user together with the various features supported by OSNs makes configuring the access control rules non-trivial. We then show how an example OSN policy can be represented in the context of our model. In order to understand the impacts of some access control configuration, we need to analyze the access control policies. Manual analysis is error-prone and tedious and may be beyond the capabilities of the non-technical user. Towards this end we use our proposal for ReBAC as a reference model to demonstrate typical misconfigurations and potential problems associated with a user's access control settings using automated techniques.

We advocate the use of Alloy [13], which supports automated analysis, for checking OSN policies. Alloy is a formal modelling language capable of expressing complex structural constraints and behaviour. Alloy is supported by an automated constraint solver called the Alloy Analyzer that searches for instances of the model that satisfy modelled system properties. The model is automatically translated into a Boolean expression, which is analysed by SAT solvers embedded within the Alloy Analyzer. A user-specified scope on the model elements bounds the domain, making it possible to create finite Boolean formulas that can be evaluated by the SAT-solver. When a property does not hold, a counter example is produced that demonstrates how it has been violated.

The rest of the paper is organized as follows. Section 2 gives a description of our relationship-based access control model. Section 3 demonstrates how we can formalize such a model in Alloy and detect potential misconfigurations in an automated manner. Section 4 concludes the paper with some pointers to future directions.

\section{REBAC MODEL}

We propose a simple UML class diagram domain model for ReBAC, and show this in Figure 1 and explain it below.

In this section, we describe the various entities and their associations in an OSN. The different entity sets are denoted by User, Subject, Group, Application, Object, and Operation. Process is defined to be a relation (henceforth referred to as an association) between these entities. Each of these entity sets and association sets are associated with a set of attributes that describe that set.

\subsection{Entity Sets in REBAC}

User: This is the set of entities who have an account in the OSN. Each user is associated with a set of attributes. Examples of attributes include identityand profile. identity gives the identity by which the user is known in the OSN.
Although most of the above attributes are defined by the user, but some are incorporated by the system. For example, User $=\{$ Jane,Tim, George, Amy $\}$.

Group: This is the set of groups in the OSN, some are user created and others exist in the system by default. Group = $\{$ Photography, Running $\}$.

Relationship: The set of relationships supported by the OSN. Specifically, these are the categories of relations on which access control are decided. It also includes the category called customized where a user can create his own relations. For example, Relationship $=\{$ Friends, Restricts, Blocks, Bans, Follows \}.

Process: The set of applications that users can install and use in the OSN. For example, Process $=\{$ Solitaire, Greeting Cards \}.

Subject: A user has several attributes and associations (these are discussed in the next section), but only some of these attributes and associations are used in making access control decisions. The set of attributes or associations of a user that determines access control to a resource is termed as Subject. For instance, a user may get access to a resource based on his identity, his relationship to the owner of the resource, membership in some groups, or by virtue of executing some applications.

In this work, the $i d$ of the user, Group, Process, and Relationship determine the access privilege and we refer to them as Subject. For example, Subject $=\{$ Running, Amy, Family $\}$.

Object: The set of resources that need protection. Attribute of an object include its type which determines the operations on the object. An object can be either a File or a Folder. Folders are containers that store the individual files. Note that, the objects may be organized in the form of a directory structure through the use of folders. For example, Object $=\left\{\right.$ georgep, georgefl, $\left.o_{1}, o_{2}\right\}$ where georgep, georgefl refer to as the personal page and friend list of George respectively, and $o_{1}$ can be a post, and $o_{2}$ may be the folder containing the post.

Operation: This is the set of actions that can be performed on objects. For example, Operation $=\{$ Tag, View, Share, Search, Comment $\}$.

\subsection{Association between Entity Sets}

Except for Ownership, ResidesIn, and OperationAllowed that are self-explanatory, we discuss the other association between entity sets below. Associations interact in subtle ways that may reveal policy misconfigurations. We use this section to introduce the associations and return to the interactions among associations in section 3.2.

Uses: This association set, Uses, connects the entity sets User to Process and it is a many to many relation. We use tuple notations to denote the individual instances of association sets. Note that, We use a tuple notation $\left(u_{i}, a_{j}\right)$ to denote instances of this association set. In other words, $\left(u_{i}, a_{j}\right) \in U$ ses. Moreover, $\left(u_{i}, a_{j}\right) \in U$ ses $\Rightarrow u_{i} \in U$ sers $\wedge$ $a_{j} \in$ Applications $\wedge a_{j} \in$ uses $\left(u_{i}\right)$. For example, Uses $=$ 


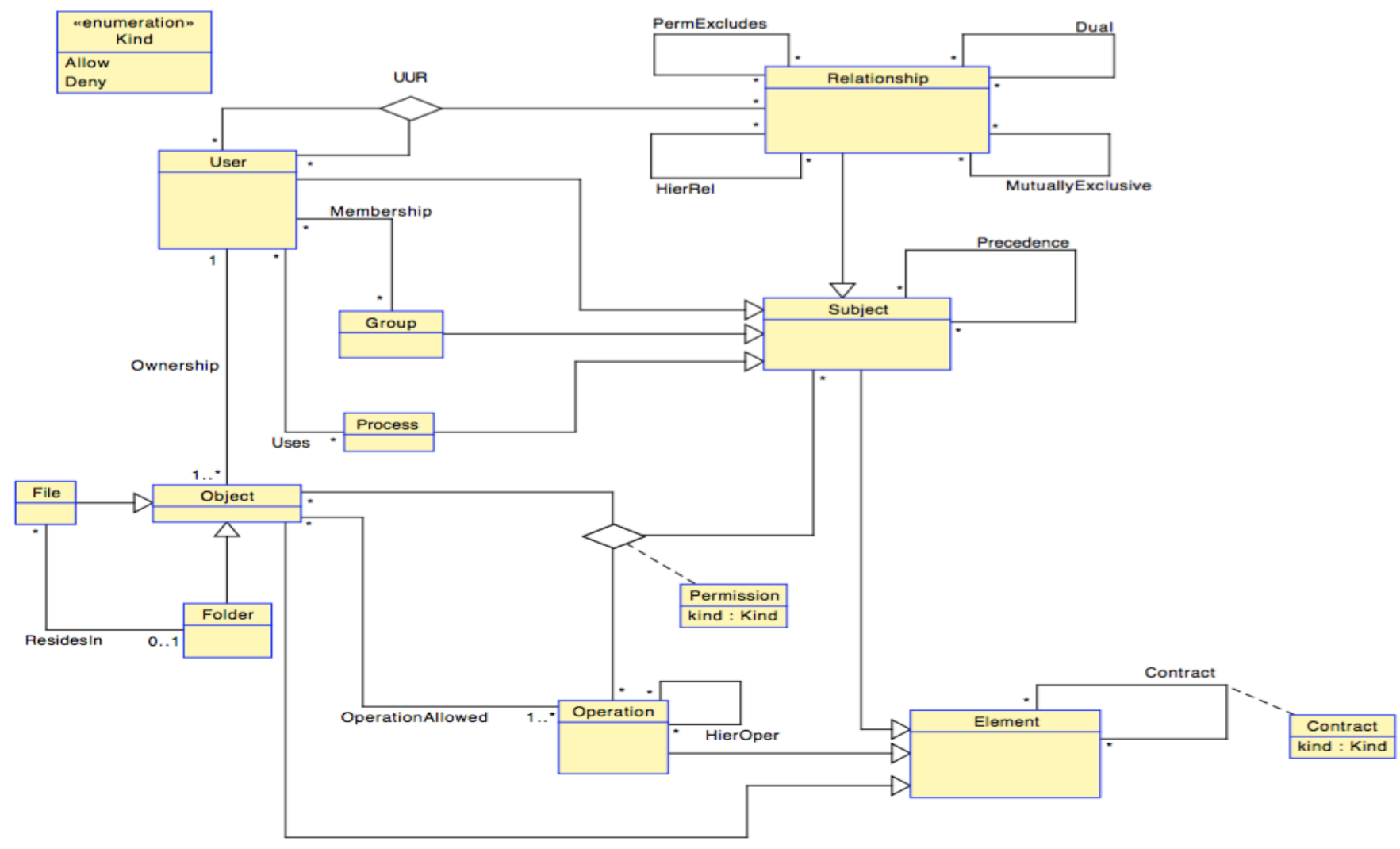

Figure 1: Simple ReBAC

$\{($ Jane, Solitaire $)\}$.

Membership: This association set, Membership, connects the entity sets Users to Groups and it has pairs of the form $\left(u_{i}, g_{m}\right)$ which indicates that the user $u_{i}$ is a member of group $g_{i} . \quad\left(u_{i}, g_{m}\right) \in$ Membership $\Rightarrow u_{i} \in U$ sers $\wedge g_{m} \in$ Groups. For example, Membership $=\{$ (George, Running), (Tim, Running) \}.

Precedence: OSNs may support both positive and negative permissions, it is possible to have conflicting permissions with respect to accessing a resource. For example, a user's group gives him access to some resource, but his identity prohibits him access on the same resource. In order to resolve such conflicts, the system defines a partial order that we term Precedence relationship which is an association over the attributes comprising the Subject. If the user's identity has a higher precedence than the group, his access will not be prohibited.

We use tuple notations $\left(s_{i}, s_{j}\right)$ to signify that $s_{i}$ has a higher precedence than $s_{j}$. For example, Precedence $=\{($ Running, Amy)\}

UUR: This association gives the user to user relation and contains a set of triples $\left(u_{i}, u_{j}, r_{m}\right)$, that specifies that user $u_{i}$ is related by $r_{m}$ to user $u_{j}$. Note that, two users $u_{i}$ and $u_{j}$ can be related by multiple relations. For example, we can have $U U R=\{$ (Jane, George, Friends), (Jane, Amy, Follows), (Amy, Jane, Bans) \}.
Dual: Each relationship has its dual. If a user $u_{i}$ is related to user $u_{j}$ with relationship $r_{m}$, then user $u_{j}$ is related to user $u_{i}$ with relationship $r_{n}$. Here the relationship $r_{m}$ is referred to as the dual of $r_{n}$ and vice-versa. This is denoted by $\left(r_{m}, r_{n}\right) \in$ Dual.

Pairs of relations that are dual of each other are specified by the system. For example, Dual $=\{($ Friends, Friends $)$, (Follows, FollowedBy)\}. The function $\operatorname{dual}\left(r_{m}\right)$ returns the dual of relationship $r_{m}$. Note that, $\operatorname{dual}\left(\operatorname{dual}\left(r_{i}\right)\right)=r_{i}$, which means that applying the dual relation twice returns the same relation.

Mutually Exclusive: This association set MutuallyExclusive gives the pairs of relations $\left(r_{i}, r_{j}\right)$ that are mutually exclusive to each other. It means that two users $u_{i}$ and $u_{j}$ cannot be related by two relations that are mutually exclusive to each other. The association is symmetric. In other words, $\left(r_{i}, r_{j}\right) \in$ MutuallyExclusive $\Longrightarrow\left(r_{j}, r_{i}\right) \in$ MutuallyExclusive. For example, MutuallyExclusive $=\{$ (Friends, Blocks), (Blocks, Follows) \}.

Hierarchy: Relations may be ordered, where the ordering relation may be referred to as HierRel which is transitive and antisymmetric and is defined by the system. For any two relations $r_{i}$ and $r_{j},\left(r_{i}, r_{j}\right) \in$ Hier Rel signifies that relation $r_{i}$ is prerequisite to relation $r_{j}$ and $r_{j}$ also gets all the positive permissions given to $r_{i}$. (Friends, Family) is an example of hierarchy. Thus, if a user $u_{k}$ can be in a relationship $r_{j}$ with $u_{m}$ only if she is in relationship $r_{i}$ with $u_{m}$. In other words, $\left(r_{i}, r_{j}\right) \in$ HierRel $\wedge\left(u_{k}, u_{m}, r_{j}\right) \in U U R \Longrightarrow\left(u_{k}, u_{m}, r_{i}\right) \in$ 
$U U R$. We use HierRel ${ }^{+}$to denote the transitive closure of HierRel. A relation $r$ and its dual cannot participate in the hierarchy relationship. Also, a relation $r$ and relations in its mutually exclusive set cannot participate in the hierarchy. Formally, $\left(r_{i}, r_{j}\right) \in \mathrm{HierRel}^{+} \Longrightarrow\left(\left(r_{i}, r_{j}\right) \notin\right.$ Dual $\wedge\left(r_{i}, r_{j}\right) \notin$ MutuallyExclusive $)$.

PermExcludes: Sometimes, we may state explicit prohibitions about when we do not want permissions to be inherited in a hierarchy. We specify this using the association PermExcludes. If $r_{i}$ is in a prerequisite relation to $r_{j}$, symbolized by $\left(r_{i}, r_{j}\right) \in$ HierRel, and we do not want $r_{j}$ to inherit the permissions of $r_{i}$, then we include $\left(r_{j}, r_{i}\right)$ in PermExcludes. That is, $\left(r_{j}, r_{i}\right) \in$ PermExcludes $\Longrightarrow\left(r_{i}, r_{j}\right) \in$ HierRel $^{+}$. The pair (Friends, Restricts) in FaceBook is an example of PermExcludes relationship.

HierOper: The operations in an OSN forms a partial order where the ordering relation is defined by the system and is referred to as HierOper. For any two operations $o p_{i}$ and $o p_{j}$, $\left(o p_{i}, o p_{j}\right) \in$ HierOper signifies that if an entity has the permission to execute $o p_{i}$ on some object $r$, then it also has the permission to execute $o p_{j}$ on $r$ provided there are no prohibitions defined on it. If the entity is prohibited from executing $o p_{i}$ on object $r$, then $o p_{j}$ may or may not be prohibited from executing $r$ depending on the type of enforcement which is discussed under contracts. For example, (View, Tag) is an example where the operations are arranged in a hierarchy.

Permissions: This is the set of allowable and prohibitable operations in a system. Each permission is an association between Subjects, Objects and Operations and has an attribute called kind which is an enumerated type having values allow and deny corresponding to positive and negative permissions respectively. Permissions may be defined by the user or by the system. Example of a permission is $\left(u_{i}, o p_{i}, o_{i}\right.$, allow) which allows user $u_{i}$ to perform operation $o p_{i}$ on object $o_{i}$. Another example is $\left(r_{j}, o p_{j}, o_{j}\right.$, deny) which prohibits relation $r_{j}$ from performing operation $o p_{j}$ on object $o_{j}$. Examples of permissions are (Amy, Share, $o_{9}$, deny) and (Solitaire, Traverse, georgefl, allow).

\subsection{Contracts}

OSNs often have contracts that specify access control rules and regulations that must be enforced. In other words, contracts are binding agreements which are imposed by the system and if violated may have legal ramifications. Contracts exist to ensure that a particular action is always allowed/disallowed regardless of the permissions set. A contract in itself is not a permission, it is a rule that governs permissions. We propose the use of contracts rather that the use of finegrained permission to express contracts because fine-grained permissions 1) would require adding a permission for every subject in the system, and 2) make the permission association more complex with respect to using a tool like the AlloyAnalyzer because the number of states that need to be considered.

Contracts can be applied a priori or a posteriori. A priori contracts are those that are enforced when a user is setting his permissions. A posteriori contracts allow a user to set his permission, however, the rules of contracts are checked when the user is trying to access some resource. Note that, $a$ posteriori may be more ideal because contracts may change with time. Thus, an a priori enforcement may not be possible if some contract has been introduced after the user has specified his access control configuration. The enforcement of contracts can be strict or soft. Strict enforcement applies not only to the element specified, but also to other elements related to it through the hierarchy. Soft enforcement, on the other hand, applies only to the element explicitly specified.

The set of contracts is specified by Contracts. Each element in this set is specified in the form of a triple $\left(e_{i}, e_{j}, p k\right)$ where $e_{i}, e_{j}$ are elements, such as, subjects, objects, and operations, and $p k$ represents the permission kind which may be allow or deny. Contracts can be specified on subjects or on objects. Contracts on subjects have the form $\left(s_{i}, o p_{i}, p k\right)$ or $\left(s_{i}, o b_{i}, p k\right)$ where $s_{i}$ is a subject, $o p_{i}$ is an operation, $o b_{i}$ is an object, and $p k$ is a permission kind. Contracts on objects have the form $\left(o b_{j}, o p_{j}, p k\right)$ where $o b_{j}$ is an object, $o p_{j}$ is an operation, and $p k$ is a permission kind. Examples of contracts are given below.

1. $\left(u_{i}, o p_{i}\right.$, allow $) \in$ Contracts which signifies that a user with user id $u_{i}$ is always allowed to perform $o p_{i}$ on any object $o_{i}$ whenever there is an element $\left(u_{i}, o p_{i}, o_{i}\right.$, allow $) \in$ Permissions.

2. $\left(g_{i}, o_{i}\right.$, deny $)$ which signifies that a user belonging to group $g_{i}$ is never allowed to perform any operation $o p_{i}$ on object $o_{i}$.

3. $\left(o_{i}, o p_{i}\right.$, allow $)$ which signifies that a subject $s_{i}$ can perform operation $o p_{i}$ on object $o_{i}$ if $\left(s_{i}, o p_{i}, o_{i}\right.$, allow $) \in$ Permissions.

The presence of contracts can further introduce a source of conflicts. Some of these may stem out of misconfigurations where what the user has specified is not what he intended. We discuss this later when dealing with misconfigurations.

\section{AUTOMATED ANALYSIS}

The presence of these various features may confuse the non-technical user. Consequently, it is important to analyze and detect potential misconfigurations in an automated manner to the extent possible. We use an Alloy model for the purpose of automated verification. We first propose a model for ReBAC expressed in Alloy, which can be instantiated for the specific OSN.

We begin by giving a brief description of Alloy. An Alloy model consists of signature declarations, fields, facts and predicates. Each signature consists of a set of atoms which are the basic entities in Alloy. Atoms are indivisible (they cannot be divided into smaller parts), immutable (their properties do not change) and uninterpreted (they do not have any inherent properties). Each field belongs to a signature and represents a relation between two or more signatures. A relation denotes a set of tuples of atoms. Facts are statements that define constraints on the elements of the model. Predicates are parameterized constraints that can be invoked from within facts or other predicates.

\subsection{Our ReBAC Model in Alloy}

We begin by defining the types for each entity set shown in Figure 1. Listing 1 shows a sample of such definitions in Alloy.

Listing 1: ReBAC Entity Sets

|| abstract sig User, Group, Relationship \{\} 
|| abstract sig File, Folder \{\}

The sets of entities involved in the ReBAC policies are then specified as shown in Listing 2 .

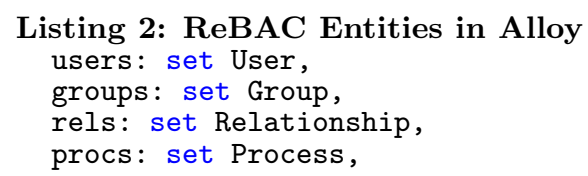

In Listing 3, we give examples of how binary, ternary, and quarternary relationships are specified in Alloy.

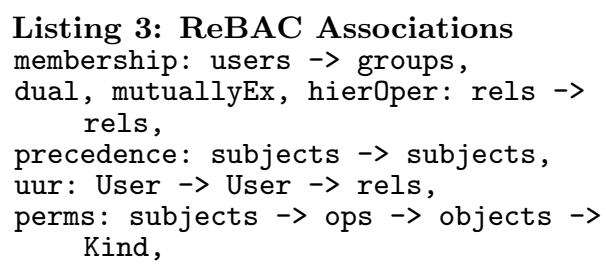

We can then specify the different types of constraints in Alloy. For enumeration type constraints, such as the set of objects are union of the set of files and folders, and the set of subjects are the sets of users, groups, rels, and procs, we show them in the form illustrated in Listing 4 . The operator + symbolizes the union operation.

\section{Listing 4: ReBAC Enumerated Type Constraints objects $=$ files + folders

Irreflexive relations are shown as follows. iden relation maps every atom to itself. \& denotes the intersection operator. In the ReBAC, a subject in a precedence relationship cannot precede itself. Similarly a relation cannot be mutually exclusive to itself. Moreover, a relation in Hierarchy cannot be present in the transitive closure of the hierarchy (denoted as `hierOper). This is illustrated in Listing 5.

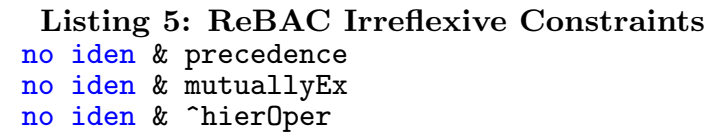

The hierarchy relation is anti-symmetric which is denoted as shown in Listing 6.

\section{Listing 6: ReBAC Anti-Symmetric Constraints || some hierOper implies !symmetric [^hierOper]}

Some relations such as dual and mutuallyEx (Mutually exclusive) are symmetric. In such cases, we just store only one pair to save resources. For instance, if $r 1 \rightarrow r 2$ is in dual, we do not store the inverse $\mathrm{r} 2 \rightarrow \mathrm{r} 1$ explicitly. It will be derived from the transpose of dual. Thus, the set of pairs of relations that are duals of each other are given by d defined as shown in Listing 7.

\section{Listing 7: ReBAC Symmetric Relations}

|| let $\mathrm{d}=$ dual $+\sim$ dual

Sometimes we may want to specify that a pair of relations specified in dual should not be an element of the set obtained by computing the transitive closure of the hierarchy. In other words, we would like to specify the disjoint properties of the sets. We demonstrate this as shown in Listing 8.

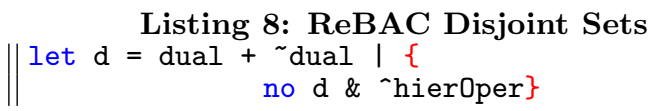

We have additional constraints that specify the nature of dual relationships as shown in Listing 9. It says that if user $u 1$ is related to $u 2$ through $r 1$, then $u 2$ is related to $u 1$ through $r 2$, where $r 2$ is the dual of $r 1$.

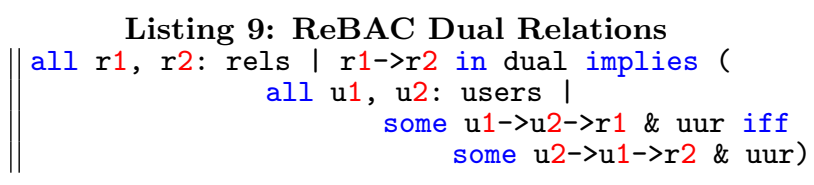

We can also add functions in Alloy for various purposes. One such example is given in Listing 10. The getPerm function uses 3 inputs, namely prms, o, and kind. prms has the same type as a permission in our model, $\circ$ is the element whose permissions we want, and kind signifies the type of permission which could be allow or deny. It produces the permissions of a particular type for a particular element. The function returns the permission associated with that element which is of the appropriate type.

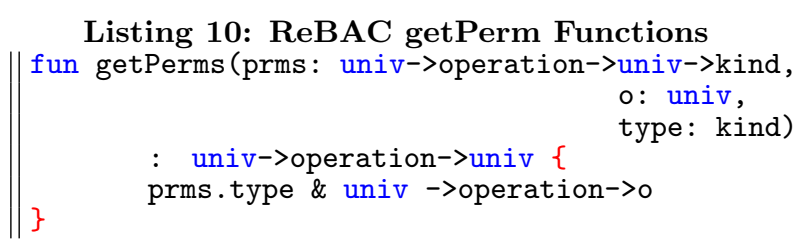

Finally, we use predicates to check whether some properties hold for the system. We may want to check whether we have conflicting permissions as shown in Listing 11 . Note that, this predicate calls the getPerm function.

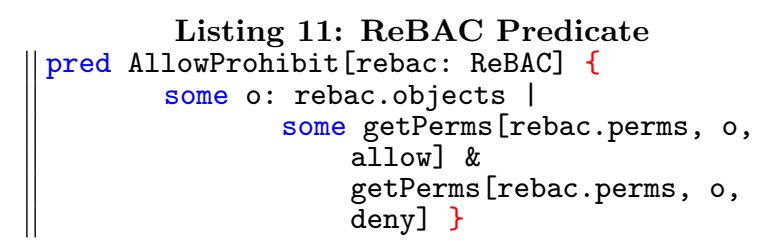

\subsection{Automated Detection of Misconfigurations}

The ReBAC Alloy model must be instantiated to detect misconfigurations in specific configurations of the OSN. We present a small example to illustrate how automated analysis can help with misconfiguration detection. We instantiate our generic model with meaningful identifiers versus the generic names that the Alloy Analyzer assigns and have the 
following policies.

User $=\{$ Jim, Mary, John $\}$

Relationships $=\{$ CloseFriends, Blocks, Friends $\}$

Operation $=\{$ View, Share, Tag, Comment, Download $\}$

Group $=\{$ Hiking $\}$

File $=\left\{o_{1}, o_{2}\right\}$

Folder $=\left\{f_{1}\right\}$

Contract $=\{($ Friends, View, Prohibit $)$, (Hiking, Share, Prohibit $)\}$

Dual $=\{($ Friends, Friends $)\}$

Hierarchy $=\{($ Friends, CloseFriends $)\}$

Membership $=\{($ Jim, Hiking $)\}$

OperationImplies $=\{($ View, Share), (Share, Tag), (Share,

Comment), (Share, Download) $\}$

OperationAllowed $=\{o \in O, o p \in O p\}$

Ownership $=\left\{\left(\mathrm{Jim}, o_{1}\right),\left(\mathrm{Jim}, f_{1}\right),(\right.$ Mary, o 2$\left.)\right\}$

Permission $=\{$ (Friends, View, o 1 , Allow $)$, (CloseFriends, Share, o $o_{1}$, Deny), (CloseFriends, View, $f_{1}$, Allow), (Mary, View, $o_{1}$, Allow)\}

Precedence $=\{($ Hiking, Friends $),($ CloseFriends, Friends $)\}$ UUR $=\{($ Jim, Mary, Friends), (Mary, Jim, Friends), (Jim, Mary, CloseFriends), (Mary, Jim, CloseFriends)\}

Listings 12 and 13 shows partial Alloy specifications indicating how this can be done. The model is instantiated with the specific users, objects, relationships, operations, etc.

Listing 12: ReBAC Instance

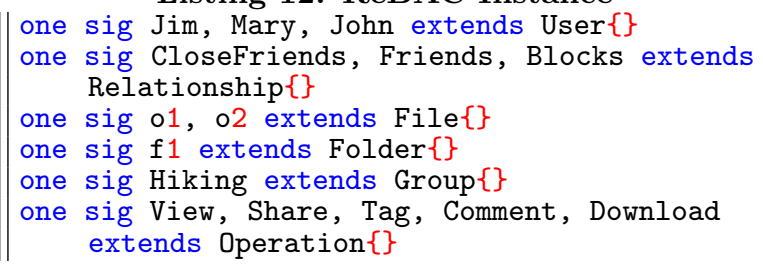

We need to specify the relationships that does not exist in the instantiated model and also augment those that does exists.

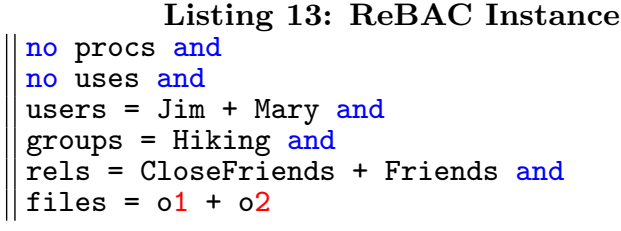

\subsubsection{Conflicting Permissions: Both Allow and Deny for the Same Object}

If Jim, who owns o1, adds the following to permissions (Friends, View, $o_{1}$, Prohibit) then we have a misconfiguration. We detect that Jim has both allowed and prohibited his Friends access to o1. Figure 2 shows this example.

We can use the Alloy code in Listing 14 on the example policy given to detect this misconfiguration. The use of the getPerms [...] function is to query the policy for permission for any object $o$ that has both Allow and Deny permissions, we then check if the permissions are for the same subject.

Listing 14: Detecting Conflicting Permissions || pred AllowProhibit [rebac: ReBAC] \{ some o: rebac.objects |

some getPerms [rebac.perms, o, Allow] \&

getPerms [rebac.perms, o, Deny] \}

\subsubsection{Contract's Effect on Permission}

In the following section, we give another example of how contracts can interfere with access control rules. Let us say that Jim makes some changes and the permissions for o1 are now as follows.

$\{($ Friends, View, o 1 , Allow $)$,

(CloseFriends, Share, $o_{1}$, Deny),

(CloseFriends, View, on , Allow)

Jim may think that members in his CloseFriends or Friends may be able to view $o_{1}$. However, if there is a contract that forbids this, the situation becomes more complex. Suppose, (Friends, View, Deny) $\in$ Contracts? In this case, the word of the contract is final and Jim may be oblivious about it. In such a scenario, members of Friends cannot view $o_{1}$.

However, we do not yet know whether members of CloseFriends will be able to view $o_{1}$. The situation is complex because of the presence of Precedence, Hierarchy, $\mathrm{Hi}$ erOper, and Strict or Soft enforcement rules. If the enforcement is strict, then members of CloseFriends should not be able to view $o_{1}$. However, for this particular example, we have (CloseFriends, Friends) $\in$ Precedence which means that CloseFriends have a higher precedence than Friends. Note that, contracts cannot override precedence relationships. Thus, in this case, members in CloseFriends will be able to view $o_{1}$. However, most users of OSNs may not be able to perform such sophisticated analysis. Consequently, it is desirable that we use automated analysis techniques to detect such problems. The counterexample produced by the Alloy Analyzer demonstrates this misconfiguration shown in Figure 3.

\section{CONCLUSION AND FUTURE WORK}

Researchers have proposed numerous models for relationshipbased access control. These models need to support a large number of operations, objects, and users connected to each other often through multiple relationships. However, the existing models fail to capture the interactions of the various operations, relationships, and the subjects. Towards this end, we propose a simple relationship-based access control model that is usable and analyzable. We express our model using the Unified Modeling Language (UML) which is the de facto standard used in the software industry and explicitly bring out the different types of associations of the different access control entities in the model.

Configuring the access control policies for such models is non-trvial. Moreover, it is very hard for the non-technical user to understand the consequences of his access control configuration. Towards this end, we demonstrate how the access control rules can be automatically checked using the Alloy Analyzer. Such analysis is needed to inform of the user of potential misconfigurations.

A lot of work remains to be done. We are aware of the limitations of Alloy Analyzer on a large instance of ReBAC and we would like to investigate ways to analyse these large instances by using a divide and conquer approach, e.g. slicing the model based on an object, relationship type, etc. We would also like to see the applicability of our relationship based access control model for other types of applications, 


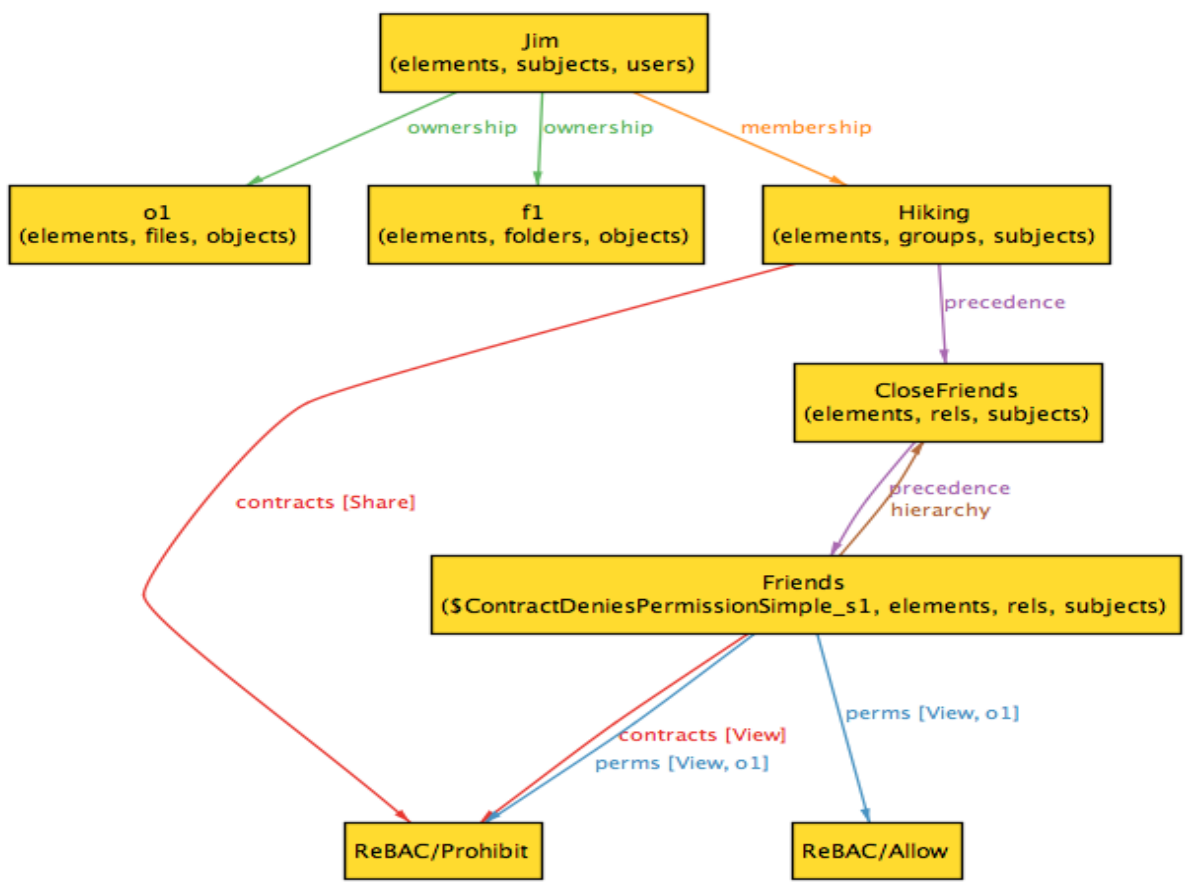

Figure 2: Misconfiguration: Conflicting Permissions (Prohibit is Deny in the text)

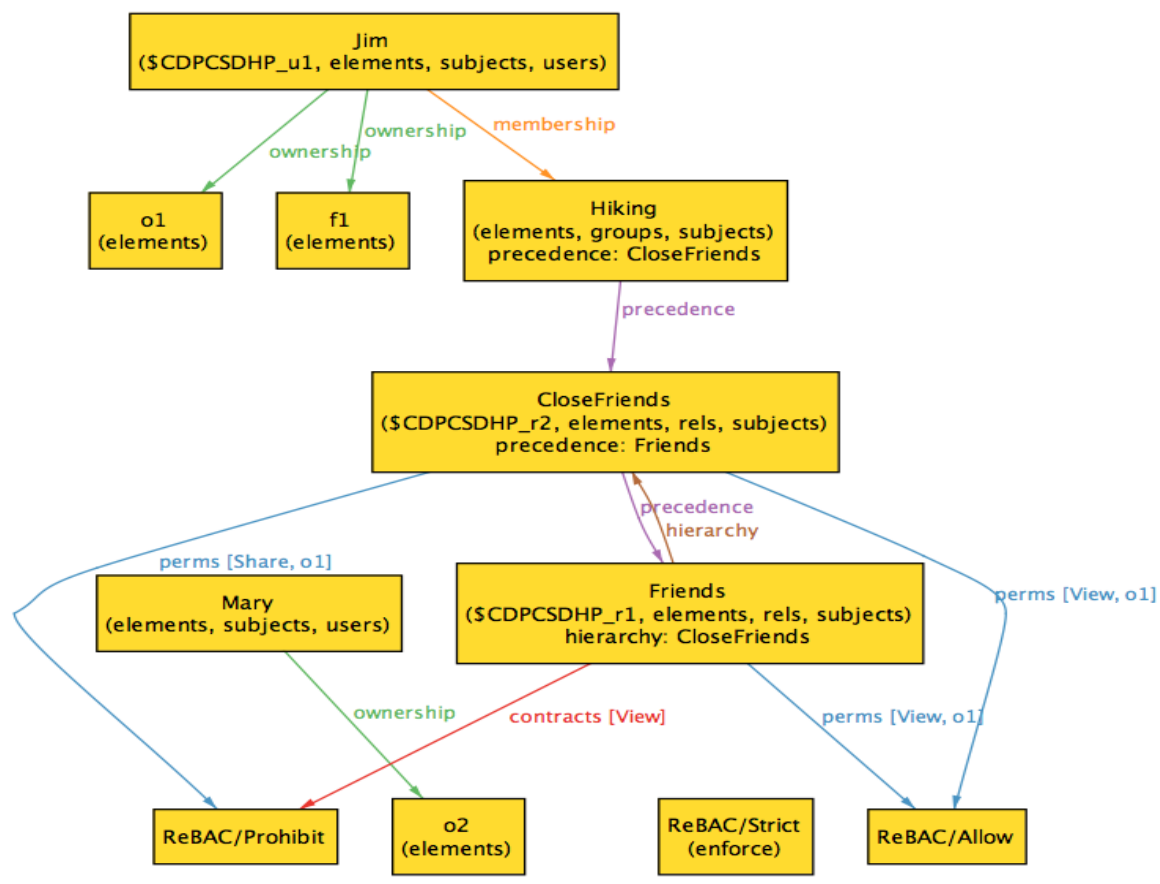

Figure 3: Misconfiguration: Complex Case where Contract Cannot Deny Permission (Prohibit is Deny in the text) 
such as, those used in the medical domain. The other work that we plan to investigate is the comparison of our model to attribute-based access control with regards to performance and expressiveness.

\section{ACKNOWLEDGEMENTS}

The research was funded in part by NIST under Grant No. 70NANB14H059, by NSF under Grant No. CCF-1018711 and by an internal grant from the Colorado State University.

\section{REFERENCES}

[1] L. Bauer, S. Garriss, and M. K. Reiter. Detecting and resolving policy misconfigurations in access-control systems. ACM Transactions on Information Systems Security, 14(1):2, May 2011.

[2] G. Bruns, P. W. L. Fong, I. Siahaan, and M. Huth. Relationship-based access control: its expression and enforcement through hybrid logic. In E. Bertino and R. S. Sandhu, editors, CODASPY, pages 117-124. ACM, 2012.

[3] B. Carminati, E. Ferrari, and A. Perego. Enforcing access control in web-based social networks. $A C M$ Transactions on Information Systems Security, 13(1), October 2009.

[4] B. Carminati, E. Ferrari, and A. Perego. Enforcing access control in web-based social networks. ACM Trans. Inf. Syst. Secur., 13(1), 2009.

[5] Y. Cheng, J. Park, and R. S. Sandhu. Relationship-based access control for online social networks: Beyond user-to-user relationships. In International Conference on Privacy, Security, Risk and Trust, PASSAT, and International Confernece on Social Computing, SocialCom, pages 646-655, Amsterdam, Netherlands, September 2012. IEEE.

[6] Y. Cheng, J. Park, and R. S. Sandhu.

Relationship-based access control for online social networks: Beyond user-to-user relationships. In SocialCom/PASSAT, pages 646-655. IEEE, 2012.

[7] Y. Cheng, J. Park, and R. S. Sandhu. A user-to-user relationship-based access control model for online social networks. In N. Cuppens-Boulahia, F. Cuppens, and J. García-Alfaro, editors, DBSec, volume 7371 of Lecture Notes in Computer Science, pages 8-24. Springer, 2012.

[8] J. Crampton and J. Sellwood. Path conditions and principal matching: a new approach to access control. In 19th ACM Symposium on Access Control Models and Technologies, SACMAT '14, London, ON, Canada - June 25 - 27, 2014, pages 187-198, 2014.

[9] M. Dammati and S. Mondal. Detecting policy misconfigurations in temporal domain using object priority. In Communication, Networks and Satellite (ComNetSat), 2012 IEEE International Conference on, pages 147-151, July 2012.

[10] T. Das, R. Bhagwan, and P. Naldurg. Baaz: A system for detecting access control misconfigurations. In USENIX Security Symposium, Security Symposium, pages 161-176, Washington DC, August 2010. USENIX Association.

[11] P. W. L. Fong. Relationship-based access control: protection model and policy language. In R. S.
Sandhu and E. Bertino, editors, CODASPY, pages 191-202. ACM, 2011.

[12] C. E. Gates. Access control requirements for web 2.0 security and privacy. In IEEE Web 2.0 privacy and security workshop (W2SP'07), California, USA, May 2007.

[13] D. Jackson. Alloy 4.0 reference manual. http://alloy.mit.edu/alloy/documentation/bookchapters/alloy-language-reference.pdf, 2012.

[14] Y. Javed and M. Shehab. Access control policy misconfiguration detection in online social networks. In International Conference on Social Computing, SocialCom, pages 544-549. IEEE, September 2013.

[15] M. L. Johnson, S. Egelman, and S. M. Bellovin. Facebook and privacy: it's complicated. In L. F. Cranor, editor, Symposium On Usable Privacy and Security , SOUPS, page 9. ACM, July 2012.

[16] M. Madejski, M. L. Johnson, and S. M. Bellovin. A study of privacy settings errors in an online social network. In IEEE International Conference on Pervasive Computing and Commonications, PerCom, Workshops, pages 340-345. IEEE, March 2012.

[17] P. Naldurg, R. Bhagwan, and T. Das. Understanding policy intent and misconfigurations from implementations: Consistency and convergence. (Short Paper) The 8th International Workshop on Formal Aspects of Security and Trust, September 2011.

[18] A. C. Squicciarini, H. Xu, and X. L. Zhang. Cope: Enabling collaborative privacy management in online social networks. Journal of the American Society for Information Science and Technology, JASIST, 62(3):521-534, March 2011.

[19] N. Zhang, M. Ryan, and D. Guelev. Evaluating access control policies through model checking. In J. Zhou, J. Lopez, R. Deng, and F. Bao, editors, Information Security, volume 3650 of Lecture Notes in Computer Science, pages 446-460. Springer Berlin Heidelberg, 2005.

[20] R. Zhang. Relation Based Access Control, volume 5 of Studies on the Semantic Web. IOS Press, 2010.

[21] R. Zhang, A. Artale, F. Giunchiglia, and B. Crispo. Using description logics in relation based access control. In B. C. Grau, I. Horrocks, B. Motik, and U. Sattler, editors, Description Logics, volume 477 of CEUR Workshop Proceedings. CEUR-WS.org, 2009.

[22] R. Zhang, F. Giunchiglia, B. Crispo, and L. Song. Relation-based access control: An access control model for context-aware computing environment. Wireless Personal Communications, 55(1):5-17, 2010. 